\title{
Lion (Panthera Leo) Feces Deter Passage of Sika Deer (Cervus Nippon)
}

\author{
Shingo Ohashi \\ Chiaki Nishi \\ Yoshitaka Deguchi \\ Hisayoshi Kofujita \\ Kazuei Matsubara \\ The United Graduate School of Agricultural Sciences \\ Iwate University \\ Japan \\ Rikio Takase \\ Takuo Murakami \\ JR-EAST Co., Ltd \\ Morioka branch \\ Japan
}

\begin{abstract}
We tested whether lion (Panthera leo) feces, the extract of lion feces, the extract of dried lion feces, and the fractions separated from the extract could have a repellent effect on sika deer (Cervus nippon). Two gates were set up in an experimental pen of sika deer and one of the two gates was treated with either lion feces, feces extract, or fractions separated from the feces extract, and the number of passages of sika deer through the gate was counted. The sika deer decreased the number of passages through the treated gates, indicating that lion feces acted as a repellent to deter the movement of the deer. Based on gas chromatography-mass spectrometry analysis, the major constituents of the most effective fraction, which was separated from the extract of dried lion feces, were fatty acids, aliphatic alcohols, aliphatic aldehydes, fatty acid amides, and steroids.
\end{abstract}

Keywords: Sika deer, Feces, Repellent, Human-wildlife interactions.

\section{Introduction}

Collisions between trains and sika deer (Cervus nippon) occur along railways in Japan, and the resources required to handle the accidents and associated damages to passengers have become a major social problem. Aside from a report on the relationship between deer-train collisions and daily activity of sika deer (Ando 2003), little research has been conducted on this issue; however, there have been a few studies on the repellent effects of carnivore odor on sika deer. Kuroda and Honda (2004) sprayed lion (Panthera leo) feces on young Japanese cypress (Chamaecyparis obtuse) to prevent feeding damage by sika deer in Yamanashi, Japan and found no damage in the experimental sites for five months. Tiger (Panthera tigris) feces temporarily reduced feeding damage by sika deer for two months in Tokushima, Japan (Kawamura et al., 2003). Ninomiya et al. (2007) showed that wolf (Canis lupus) feces suppressed feeding behavior, and induced aversive behavioral responses in sika deer. However, in these studies, the specific compound that controls the behavior of sika deer was not investigated.

The analysis of volatile components contained in urine and other secretions from predators has been performed in wolf (Raymer et al., 1986), bobcat (Lynx rufus) (Mattina et al., 1991), lion (Andersen and Vulpius, 1999), cheetah (Acinonyx jubatus) (Burger et al., 2006), male Bengal tiger (Burger et al., 2008), anal sacs of dog (Canis familiaris) and coyote (Canis latrans)(Preti et al., 1976), anal glands of wolverine (Gulo gulo) (Wood et al., 2005), and subcaudal gland secretions of European badger (Meles meles) (Buesching et al., 2002), but these experiments did not test the repellent effects of components. 
The odors of excretions and secretions of predators have been used to induce feeding avoidance in prey animals (Arnould et al., 1998), but tests of the efficacy of these odors to deter the intrusion or passage of prey animals to a specific area have not been conducted. The identification of repellent chemicals may be an important contribution to the prevention of collisions between trains and sika deer. Based on previous research with lion feces, it was hypothesized that the lion feces had a repellent effect on sika deer, and the effect may be active for five months. The persistent activity was probably due to the release of feces odor over a long time period, or the active components may not be highly volatile chemicals found in raw feces. The presence of high molecular weight chemicals in feces are more stable and will remain unaltered in feces for longer times than other highly volatile compounds found in the anal-sac or the urine (Martin et al., 2010). In this study, we tested the repellent effect of lion feces, feces extracts, and the extract of dried lion feces on captive sika deer by presenting the sample and measuring the passage of deer through an experimental gate. Second, we separated the extracts of dried lion feces into four fractions, and partially analyzed the constituents by gas chromatography-mass spectrometry (GC-MS). Further, the synthetic mixture of volatile constituents was tested to assess if it had the repellent effect.

\section{Materials and methods}

\subsection{Animals}

Feces were collected from three adult lions (one male and two females) which was bred in Morioka City Zoological Park, Morioka, Japan. Lions fed $84 \mathrm{~kg}$ of beef, $35 \mathrm{~kg}$ of beef bones, and supplements each week, and were fasted once per week; daily feeding dosage was varied according to their conditions. The repellent effect of lion feces was assessed on six sika deer (one male and five females) in captivity in Morioka City Zoological Park using sample presentation tests (bioassays). The exhibition time at Morioka City Zoological Park was 9:00 to 16:30, and the sika deer were fed pellets, hay cubes, and straw on the feeding floor after the exhibition time. Water and salt were fed ad libitum.

\subsection{Fecal Samples}

Lion feces were collected from the lion breeding floor and stored at $4^{\circ} \mathrm{C}$. The feces were dried in the sun for about two weeks then crushed with a blender and stored at $4^{\circ} \mathrm{C}$ until extraction with desiccant agent (silica gel). The crushed feces were agitated with diethyl ether, stirred in a beaker, and stored at $4{ }^{\circ} \mathrm{C}$ overnight. Suction filtration was used to collect the filtrate, and the solvent was removed in a rotary evaporator. Deer feces were collected from the deer breeding floor and stored at $4^{\circ} \mathrm{C}$. An extract was obtained from the deer feces using the same method as described above.

To prepare the sample, $100 \mathrm{mg}$ aliquots of the concentrated extract of dried lion feces were dissolved in $1 \mathrm{ml}$ of ethanol by sonication, and stored in a glass vial. Fractionation was performed with a Lobar column (Size B, 310 mm, 25 mm ID, Li Chroprep RP-18, 40-63 $\mu \mathrm{m}$, Merck). The column was equipped with an RI detector (RI-10, EYELA) and medium pressure pump (VSP-3050, EYELA). Flow rate of the ethanol as the eluting solvent was 5 $\mathrm{ml} / \mathrm{min}$. The extract was separated and collected into four fractions (Fr.1, 2, 3, and 4) corresponding to four peaks obtained by the RI detector. The solvent was removed in a rotary evaporator.

\subsection{Experimental Pen in Bioassay}

All bioassays were performed in the sika deer pen in Morioka City Zoological Park. The pen had an area of 225 $\mathrm{m} 2$, and some trees and rocks were scattered throughout the area. After the daily exhibition time, a net ( $2 \mathrm{~m}$ in height and $10 \mathrm{~m}$ in width) was set transversely between two trees located at each end about $1.5 \mathrm{~m}$ from the center of the pen. This net separated the pen into two compartments and restricted the movement of sika deer through the gates at the ends of the net (Figure 1). A pair of poly-vinyl-chloride pipes ( $1 \mathrm{~m} \mathrm{long}$ and $4 \mathrm{~cm}$ in outer diameter) was set in both gates. Behavior of sika deer around both gates was recorded every day by infrared cameras (WVCD820, Panasonic). 


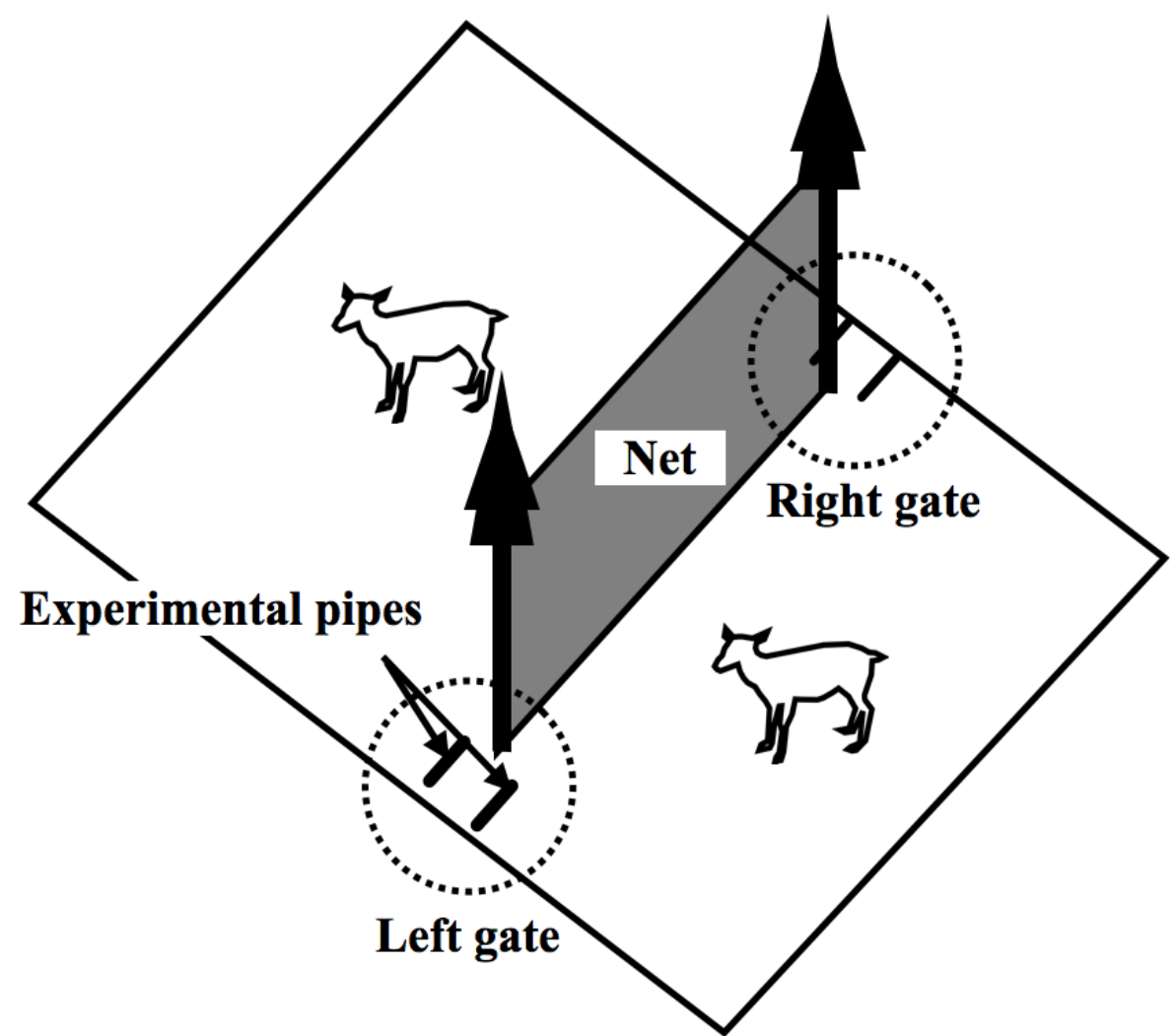

Figure 1. Experimental pen of sika deer in Morioka Zoological Park.

Samples were applied to the experimental pipes in one gate, and the number of passages of sika deer through both the right and left gates was counted by using video tape recorder.

\subsection{Sample Presentation in Bioassay}

Each concentrated sample derived from $100 \mathrm{~g}$ of dried feces was dissolved in $100 \mathrm{ml}$ of ethanol and stored at $20^{\circ} \mathrm{C}$ until used in the bioassay. Samples used in the assays included $5 \mathrm{~g}$ of raw lion feces (RLF), $5 \mathrm{ml}$ of the extract of raw lion feces (E-RLF), the extract of dried lion feces (E-DLF), Fr.1 to Fr.4 separated from E-DLF, and ethanol as a control. These samples were diluted to $500 \mathrm{ml}$ aqueous solution and soaked into Kim- towels (NIPPON PAPER). Ten holes were made at regular intervals within the experimental pipes so that the odor would spread easily. Samples were packed into the experimental pipes, and the pipes were fixed with lids at both ends. New clean pipes were used for each sample.

The experimental period consisted of three days preparation and four days sample presentation. A sample was set into the experimental pipes on one gate (treatment gate) and empty pipes were set on the other gate (control gate) during the sample presentation period. The conditioning period, when no sample was presented, lasted for one week after each experimental period to prevent the previous sample from influencing the next experiment.

\subsection{Behavioral Evaluation}

The behavior of sika deer around the gates during the experimental period was recorded by infrared camera and the number of passages through the gates was counted. The behavior of the sika deer was recorded for about 16.5 hours from 16:30 to 9:00. The $\chi$-square test was performed between the observed value and the expected value; the observed value is mean number of passages observed in the sample presentation period, the expected value is mean number of passages calculated from the total number of passages multiplied by the ratio of the number of passages of the right and left gates during the preparation period. It was estimated that sika deer avoided the sample when the number of passages through the experimental gate in the experimental period (observed value) was significantly larger than those in the control period (expected value), and the $\chi$-square value was positively associated with the size of the effect.

\subsection{GC Analyses}


E-DLF and Fr.1 to Fr.4 were used in the GC analysis. Concentrated fecal samples (10 mg) were dissolved in $1 \mathrm{ml}$ of hexane (n-hexane). GC was performed with a GC-1700 (Shimadzu) equipped with a capillary column (MDN$5 \mathrm{~S}, 30 \mathrm{~m}, 0.25 \mathrm{~mm} \mathrm{ID}$, SUPELCO). Nitrogen was used as the carrier gas at a flow rate of $1 \mathrm{ml} / \mathrm{min}$. The temperature of the injector was set at $300^{\circ} \mathrm{C}$, and the split ratio was set at 1:20. The oven temperature was programmed at $120^{\circ} \mathrm{C}$ for $10 \mathrm{~min}$, then increased by $3^{\circ} \mathrm{C} / \mathrm{min}$ to $300^{\circ} \mathrm{C}$, and finally held at $300^{\circ} \mathrm{C}$ for $20 \mathrm{~min}$. The flame ionization detector was used at a temperature of $200^{\circ} \mathrm{C}$.

\subsection{GC-MS Analyses}

Fr.2, Fr.3, and the extract of deer feces were used in the GC-MS analysis. Concentrated samples (10 mg) were dissolved in $1 \mathrm{ml}$ of hexane (n-hexane). GC-MS was performed with a GCMS-QP2010 (Shimadzu) equipped with a capillary column (MDN-5S, $30 \mathrm{~m}, 0.25 \mathrm{~mm}$ ID, SUPELCO). Helium was used as the carrier gas at a flow rate of $1 \mathrm{ml} / \mathrm{min}$. The temperature of the injector was set at $300^{\circ} \mathrm{C}$, and the split ratio was set at 1:20. The oven temperature was programmed at $120^{\circ} \mathrm{C}$ for $10 \mathrm{~min}$, then increased by $3^{\circ} \mathrm{C} / \mathrm{min}$ to $300^{\circ} \mathrm{C}$, and finally held at $300^{\circ} \mathrm{C}$ for $20 \mathrm{~min}$. Electron impact was used for ionization, which was set at $70 \mathrm{eV}$ and $200^{\circ} \mathrm{C}$. Mass spectral fragments below $\mathrm{m} / \mathrm{z}=39$ were not recorded. The relative amount of each component was determined as the percent of the total ion current (TIC).

Initial identification of compounds was achieved by comparison of their electron impact (EI) mass spectra with mass spectra from the mass spectral library Wiley 7th. When possible, identifications were confirmed by comparison of spectra and retention times with those of authentic standards (from Sigma-Aldrich Chemical Co., Tokyo Chemical Industry Co., Wako Pure Chemical Industries, Kanto Chemical Co.). The retention time and the mass spectra of the peaks detected with deer feces were compared with the lion feces.

The identified compounds were classified in each compound group, which were fatty acids, aliphatic alcohols, aliphatic aldehydes, fatty acid amides, and steroids. To mimic Fr.2, eight chemicals; 9-octadecenoic acid and hexadecanoic acid as fatty acids, pentadecanol as aliphatic alcohols, tridecanal as aliphatic aldehydes, 9octadecenamide as fatty acid amides, and cholesterol and rosterone as steroids were mixed. Each compound was mixed to the proportions of each compound group in $10 \mathrm{mg}$ of Fr.2 and dissolved in $5 \mathrm{ml}$ of ethanol. After that, the repellent effect of the mixture was tested by the bioassay.

\section{Results}

\subsection{Behavioral Evaluation}

The results of the bioassay indicated that sika deer significantly avoided RLF ( $\mathrm{P}<0.001)$, E-RLF $(\mathrm{P}<0.001)$, and E-DLF ( $\mathrm{P}<0.001)$, but did not avoid ethanol as the control (Table 1). Similarly, significant differences were found in the number of passages when all fractions were presented to sika deer $(\mathrm{P}<0.05$ or $\mathrm{P}<0.001)$ (Table 2$)$. In addition, the $\chi$-square value was the highest when Fr. 2 was presented to sika deer at the right gate $(\chi 2=256.27$, $\mathrm{P}<0.001)$, and the second highest value was Fr.3 at the left gate $(\chi 2=153.64, \mathrm{P}<0.001)$, whereas Fr.4 was the lowest at the right gate $\left(\chi^{2}=4.76, \mathrm{P}<0.05\right)$. The average $\chi$-square value of the right and left gates was the highest when Fr.2 was presented $\left(\chi^{2}=163.66\right)$. The highest $\chi$ square value meant that the passage of the deer was inhibited most. From comparison of the $\chi$ square values when these fractions were presented, the rank order of the relative strength was Fr. $2>$ Fr. $3>$ Fr. $4>$ Fr.1, and Fr. 2 was thought to be the most effective fraction. Although a significant difference was found in the number of passages when the synthetic mixture of eight chemicals was used (Table 2), it did not seem to have repellent effect because deer passed through the control gate more than experimental gate on the third day of sample presentation (data not shown). 
Table 1. Comparison of the repellent effect of raw lion feces, extract of raw lion feces, extract of dried lion feces and ethanol

\begin{tabular}{|c|c|c|c|c|c|c|}
\hline \multirow{2}{*}{\multicolumn{2}{|c|}{ Sample }} & \multirow{2}{*}{$\begin{array}{l}\text { Treatme } \\
\text { nt gate }\end{array}$} & \multicolumn{3}{|c|}{$\begin{array}{l}\text { The mean of the number of passages in } \\
\text { experimental period }\end{array}$} & \multirow{2}{*}{$\chi^{2}$ value $^{c}$} \\
\hline & & & Gate & $\begin{array}{l}\text { Expected } \\
\text { value }^{a}\end{array}$ & $\begin{array}{l}\text { Observed } \\
\text { value }^{b}\end{array}$ & \\
\hline \multirow{2}{*}{\multicolumn{2}{|c|}{ Raw Lion Feces }} & \multirow{2}{*}{ Left } & Right & 49.2 & 80.2 & \multirow{2}{*}{$50.59^{*}$} \\
\hline & & & Left & 31.0 & 0.0 & \\
\hline \multirow{2}{*}{$\begin{array}{l}\text { Extract of Raw } \\
\text { Feces }\end{array}$} & \multirow{2}{*}{ Lion } & \multirow{2}{*}{ Left } & Right & 43.9 & 77.8 & \multirow{2}{*}{$59.53 *$} \\
\hline & & & Left & 34.4 & 0.5 & \\
\hline \multirow{2}{*}{$\begin{array}{l}\text { Extract of Dried } \\
\text { Feces }\end{array}$} & Lion & \multirow{2}{*}{ Left } & Right & 44.6 & 100.7 & \multirow{2}{*}{$126.26^{*}$} \\
\hline & & & Left & 56.6 & 0.5 & \\
\hline \multirow{2}{*}{\multicolumn{2}{|c|}{$\begin{array}{l}\text { Ethanol } \\
\text { (control) }\end{array}$}} & \multirow{2}{*}{ Left } & Right & 56.2 & 53.1 & \multirow{2}{*}{$0.52 *$} \\
\hline & & & Left & 26.4 & 29.4 & \\
\hline
\end{tabular}

${ }^{\mathrm{a}}$ The mean of the total number of passages in the sample presentation period was multiplied by the ratio of the number of passages of right and left gate during the preparation period.

$\mathrm{b}$ The mean of the number of passages observed in sample presentation period.

${ }^{c}$ Chi-square test was conducted on the number of passages by comparison of the observed value with the expected value $(\mathrm{df}=1){ }^{*}: \mathrm{P}<0.001(\chi 2>10.828)$ indicates significant reduction of the number of passages.

Table 2. Comparison of the repellent effect of fractions separated from extract of dried lion feces and synthetic mixture on the number of passages of sika deer

\begin{tabular}{|c|c|c|c|c|c|c|}
\hline \multirow{2}{*}{ Sample } & \multirow{2}{*}{$\begin{array}{l}\text { Treatment } \\
\text { gate }\end{array}$} & \multicolumn{3}{|c|}{$\begin{array}{l}\text { The mean of the number of } \\
\text { passages in experimental period }\end{array}$} & \multirow{2}{*}{$\chi^{2}$ value $^{c}$} & \multirow{2}{*}{$\begin{array}{l}\text { The mean of } \\
\chi^{2} \text { value }\end{array}$} \\
\hline & & Gate & $\begin{array}{l}\text { Expected } \\
\text { value }^{a}\end{array}$ & $\begin{array}{l}\text { Observed } \\
\text { value }^{b}\end{array}$ & & \\
\hline \multirow{4}{*}{ Fr.1 } & \multirow{2}{*}{ Right } & Right & 46.9 & 22.3 & \multirow{2}{*}{$32.16 * *$} & \multirow{4}{*}{31.4} \\
\hline & & Left & 31.6 & 56.3 & & \\
\hline & \multirow{2}{*}{ Left } & Right & 66.6 & 98.5 & \multirow{2}{*}{$30.58 * *$} & \\
\hline & & Left & 67.0 & 35.0 & & \\
\hline \multirow{4}{*}{ Fr. 2} & \multirow{2}{*}{ Right } & Right & 69.9 & 4.5 & \multirow{2}{*}{$256.27 * *$} & \multirow{4}{*}{163.7} \\
\hline & & Left & 21.9 & 87.3 & & \\
\hline & \multirow{2}{*}{ Left } & Right & 34.6 & 70.0 & \multirow{2}{*}{$71.05 * *$} & \\
\hline & & Left & 35.9 & 0.5 & & \\
\hline \multirow{4}{*}{ Fr.3 } & \multirow{2}{*}{ Right } & Right & 41.3 & 20.8 & \multirow{2}{*}{$39.06^{* *}$} & \multirow{4}{*}{96.4} \\
\hline & & Left & 68.8 & 89.3 & & \\
\hline & \multirow{2}{*}{ Left } & Right & 42.3 & 106.0 & \multirow{2}{*}{$153.64 * *$} & \\
\hline & & Left & 70.5 & 6.8 & & \\
\hline \multirow{4}{*}{ Fr.4 } & \multirow{2}{*}{ Right } & Right & 204.0 & 184.3 & \multirow{2}{*}{$5.76^{*}$} & \multirow{4}{*}{63.1} \\
\hline & & Left & 101.7 & 121.5 & & \\
\hline & \multirow{2}{*}{ Left } & Right & 76.0 & 122.5 & \multirow{2}{*}{$57.33 * *$} & \\
\hline & & Left & 74.7 & 28.3 & & \\
\hline \multirow{2}{*}{$\begin{array}{l}\text { Synthetic } \\
\text { mixture }\end{array}$} & \multirow{2}{*}{ Left } & Right & 50.1 & 68.0 & \multirow{2}{*}{$15.09 * *$} & \multirow{2}{*}{-} \\
\hline & & Left & 36.6 & 18.8 & & \\
\hline
\end{tabular}


${ }^{a}$ The mean of the total number of passages in the sample presentation period was multiplied by the ratio of the number of passages of right and left gate during the preparation period. $\mathrm{b}$ The mean of the number of passages observed in sample presentation period. ${ }^{c}$ Chi-square test was conducted on the number of passages by comparison of the observed value with the expected value $(\mathrm{df}=1){ }^{*}: \mathrm{P}<0.05(\chi 2>3.841),{ }^{* *}: \mathrm{P}<0.001\left(\chi^{2}>10.828\right)$ indicates significant reduction of the number of passages.

Table 3. Main constituents in fraction 2 separated from extract of dried lion feces

\begin{tabular}{|c|c|c|}
\hline $\begin{array}{l}\text { eak number in } \\
\text { Fig. } 3\end{array}$ & Compound & $\%$ area of TIC \\
\hline 1 & Pentadecanal & 0.26 \\
\hline 2 & Tetradecanoic acid & 0.49 \\
\hline 3 & Hexadecanal & 0.50 \\
\hline 4 & Pentadecanoic acid & 0.43 \\
\hline 5 & 1-Hexadecanol & 0.33 \\
\hline 6 & Heptadecanal & 4.68 \\
\hline 7 & 9-Hexadecenoic acid & 1.18 \\
\hline 8 & Hexadecanoic acid & 18.31 \\
\hline 9 & 1-Heptadecanol & 0.55 \\
\hline 10 & Octadecanal & 0.54 \\
\hline 11 & 9-Octadecen-1-ol & 0.36 \\
\hline 12 & Heptadecanoic acid & 0.30 \\
\hline 13 & 1-Octadecanol & 1.69 \\
\hline 14 & 9-Octadecenoic acid & 33.38 \\
\hline 15 & 1-Nonadecanol & 6.71 \\
\hline 16 & Octadecanoic acid & 14.15 \\
\hline 17 & Hexadecanamide & 1.03 \\
\hline 18 & 1-Eicosanol & 1.53 \\
\hline 19 & 9-Octadecenamide & 1.00 \\
\hline 20 & Octadecanamide & 0.58 \\
\hline 21 & N.I. ${ }^{a}$ & 0.95 \\
\hline 22 & N.I. & 1.49 \\
\hline 23 & N.I. & 1.15 \\
\hline 24 & 12-hydroxy-Androstane-3,17-dione ${ }^{b}$ & 0.32 \\
\hline 25 & N.I. & 0.72 \\
\hline 26 & Cholesterol & 0.33 \\
\hline 27 & N.I. & 2.79 \\
\hline 28 & Cholest-4-ene-3,6-dione ${ }^{b}$ & 1.84 \\
\hline 29 & Cholest-3,6-dione $^{b}$ & 2.41 \\
\hline
\end{tabular}

\subsection{Chemical Analyses}


E-DLF was separated into four fractions corresponding to four peaks. The dry weight of each fraction were $70 \mathrm{mg}$ (5.8\%) of Fr.1, $380 \mathrm{mg}$ (31.4\%) of Fr.2, $32 \mathrm{mg}$ (26.0\%) of Fr.3 and $10 \mathrm{mg}$ (8.2\%) of Fr.4 from $1210 \mathrm{mg}$ of the extract. Comparisons of each chromatogram obtained by GC indicated that some characteristic peaks were found in the chromatogram of E-DLF and Fr.1 to Fr.4 (Figure 2).

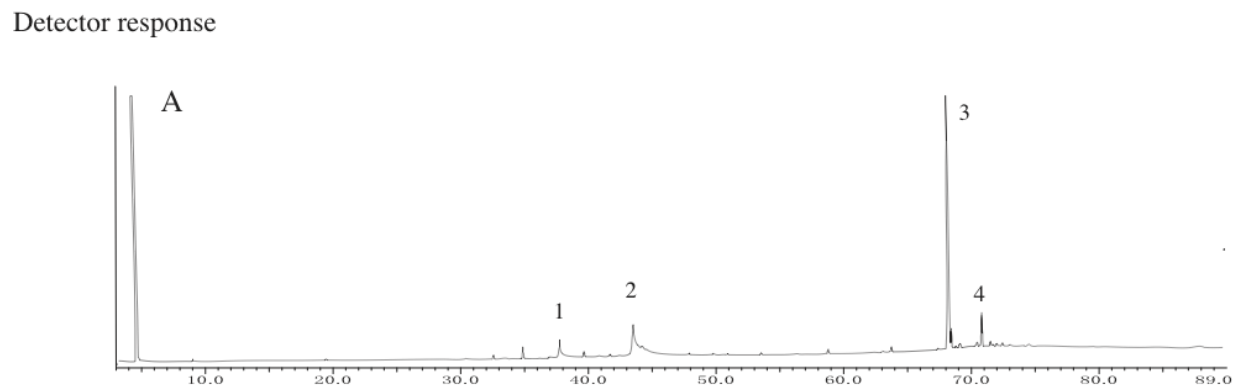

B
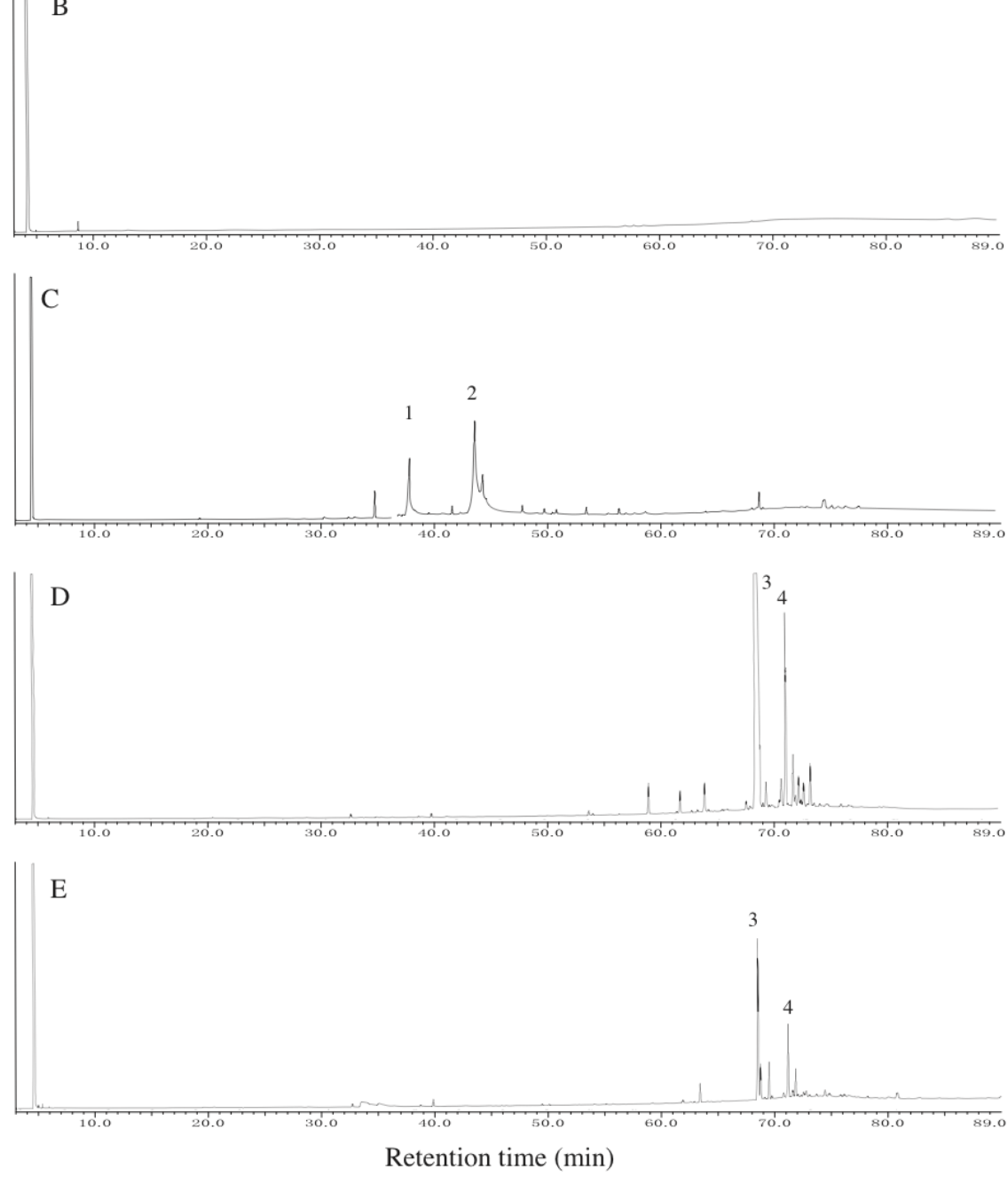

Figure 2. Comparison of chromatogram of extract of dried lion feces (E-DLF) and fractions (Fr.1 to 4) obtained by GC. A: E-DLF, B: Fr.1, C: Fr.2, D: Fr.3 and E: Fr.4. Characteristic peaks 1 and 2 were found in E-DLF and Fr.2, and peaks 3 and 4 were found in E-DLF, Fr.3 and Fr.4.

Characteristic large peaks 1, 2, 3, and 4 were found in the chromatogram of E-DLF (Figure 2A). Fraction 2 had a particularly large peak 1 and 2 (Figure 2C), and Fr.3 and Fr.4 had both peaks 3 and 4 (Figure 2D and E).

From the GC-MS analysis, major compounds in Fr.2, which had the strongest repellent effect, were identified (Figure 3, Table 3). The representative constituents contained in this fraction were composed of fatty acids with straight chains from $\mathrm{C} 14$ to $\mathrm{C} 18$ as major constituents. The proportion of each compound group were 
approximately $68.2 \%$ of fatty acids, $11.2 \%$ of aliphatic alcohols, $6.0 \%$ of aliphatic aldehydes, $2.6 \%$ of fatty acid amides, and $4.9 \%$ of steroids. Peaks number 1 and 2 in Figure 2 were identified as hexadecanoic acid and 9octadecenoic acid. Five compounds that occupied approximately $6.1 \%$ of TIC could not be identified by the spectral analysis in this study.
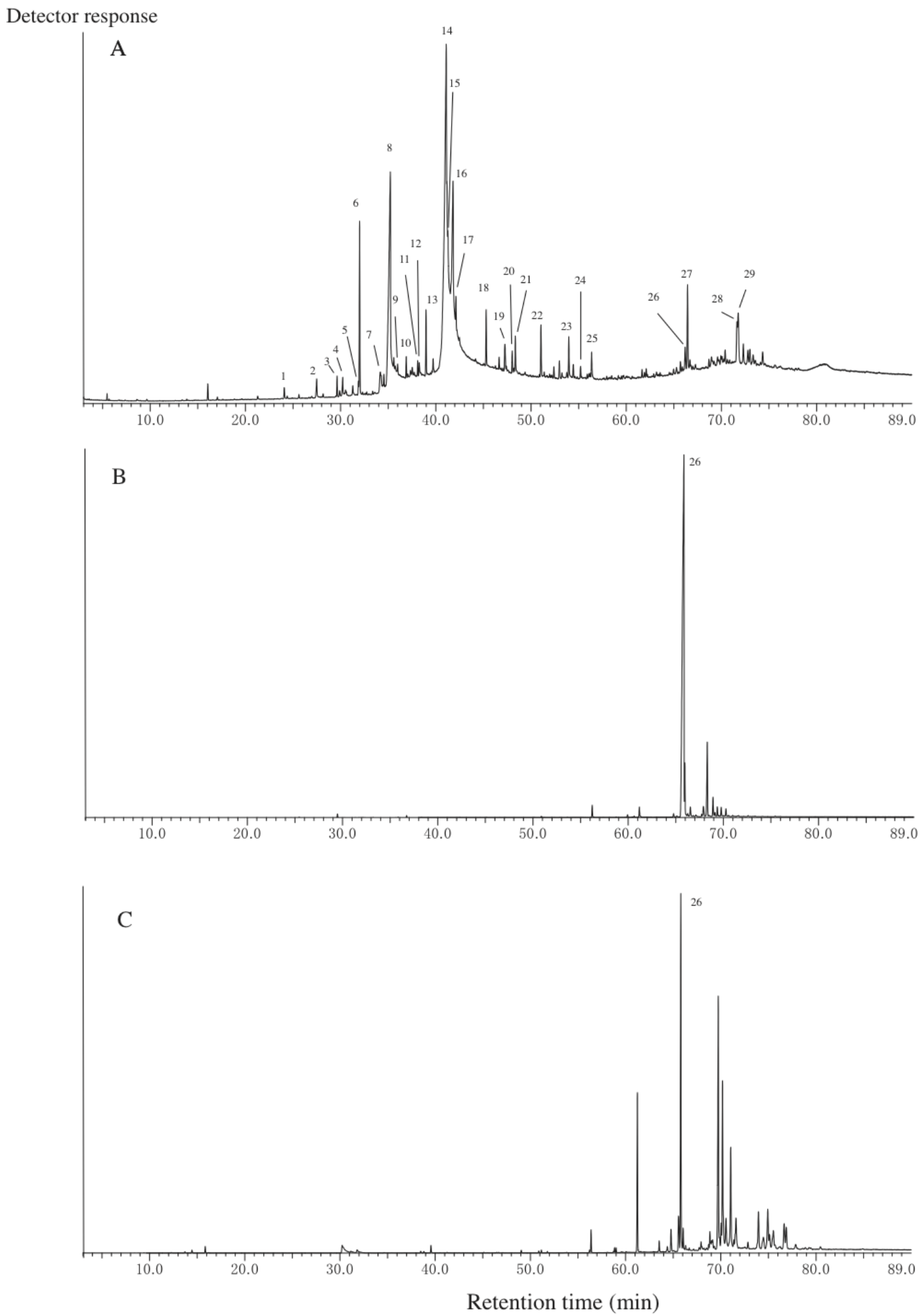
Figure 3. Comparison of the total ion chromatogram of Fr.2 (A) and Fr.3 (B) from extract of dried lion feces and the extract of dried deer feces (C) obtained by GC-MS.

The GC-MS analysis of Fr.3 showed that the constituents contained in this fraction were composed of cholesterol as major constituent, unidentified steroids, and high molecular weight hydrocarbons.

The characteristic large peak of Fr.3 (peak 26 in Figure 3B), which was peak number 3 in Figure 2, was identified as cholesterol but the peak number 4 in Figure 2 could not identified. As a result of GC-MS of dried deer feces, cholesterol was the only similar compound in comparison with lion feces (peak 26 in Figure 3C).

\section{Discussion}

Results from this study indicated that sika deer avoided lion feces. In addition, sika deer showed similar avoidance behaviors to dried lion feces and diethyl ether extracts of raw and dried lion feces. The repellent effect of lion feces on sheep (Ovis ares), rabbit (Oryctolagus cuniculus), goat (Capra hircus) and beaver (Castor canadensis) has been investigated in other studies (Arnould and Signoret, 1993; Boag and Mlotkiewicz., 1994; Weldon et al., 1993; Engelhart and Muller-Schwarze, 1995). Although these studies indicated that lion feces have a repellent effect on some herbivores, advanced chemical analysis was not conducted until the present study. Sullivan et al. (1985) reported that feces and urine of some carnivore animals effectively suppressed feeding by black-tailed deer (Odocoileus hemionus columbianus) in a pen bioassay. They also reported that coyote urine provided the most consistent suppression. Swihart et al. (1991) reported that urine of bobcat and coyote reduced browsing by white-tailed deer (Odocoileus virginianus), and the extract of bobcat urine was as effective as unextracted urine. These studies indicate that some species of deer avoid the odor derived from feces or urine of carnivore animals. Seamans et al. (2002) reported that white-tailed deer did not avoid coyote hair when it was present on established deer trails. In our experiment, sika deer significantly avoided the gate treated with lion feces. This result suggests that lion feces could act as a strong deterrent to the passage of sika deer. The individual fractions separated from the extract of dried lion feces were also found to significantly deter the passage of sika deer. By comparing $\chi$-square values, it was suggested that Fr.2 had the highest repellent effect relative to the other fractions, and the compounds that repel sika deer might be contained in Fr.2.

The extract of dried lion feces and Fr.1 to Fr. 4 were used in the GC analysis, and Fr.2 and Fr.3 were also analyzed by GC-MS. A few specific peaks were detected in each fraction when the chromatograms obtained by GC were compared. This means that the constituents of the extract of lion feces were divided into Fr.1 to Fr.4. Peaks 1 and 2 in Figure 2, which were quantitatively major constituents of the extract of lion feces, were associated with Fr.2, and similarly peaks 3 and 4 were associated with Fr.3 and Fr.4. Peaks 1 and 2 in Figure 2 were identified as hexadecanoic acid and 9-octadecenoic acid. As a result of GCMS, peak 3 in Figure 2 was identified as cholesterol, but peak 4 was not be identified. Results of the GC-MC analysis estimated relatively large amounts of fatty acids and aliphatic alcohols in Fr.2. It is unlikely that only these characteristic compounds of Fr. 2 are active repellent chemicals because sika deer avoided not only Fr.2 but also other fractions. However, it was found that hexadecanoic acid, 9-octadecenoic acid, and cholesterol were included as major constituents of lion feces. Hexadecanoic acid, 9-octadecenoic acid, and cholesterol were found in feces and the urine of other carnivore animals, and other fatty acids, aliphatic alcohols, and aliphatic aldehydes were also identified (Mattina et al., 1991; Arnould et al., 1998; Buesching et al., 2002a; Wood et al., 2005; Burger et al., 2006; Burger et al 2008; Martin et al., 2010). Because these odorous constituents remained in the dried feces, it was thought that these constituents were associated with the long-term release of the fecal odor.

The comparison of the results of GC-MS analysis between the dried lion feces and the dried deer feces showed that cholesterol was the only common compound. The highly volatile compounds were identified in metatarsal glands of sika deer (Wood, 2003) and the odor of fecal pellets of rabbit (Goodrich et al., 1981). The highly volatile compounds in the deer feces were thought to be lost during the desiccation process, as these compounds might be more volatile than those in the lion feces. It was assumed that the constituents in the feces of the carnivore animals have greater potential for long-term release of fecal odor than the herbivore animals. There was no repellent effect when the mixture of eight chemicals, the imitation of Fr.2, was presented to deer. Arnould et al. (1998) reported that a synthetic mixture of mostly fatty acids and indole had a significant repellent effect on sheep, and the effect was lower than the total extract of dog feces. The simple combination of these chemicals might not be the critical source of the repellent effect. 
It might be necessary to blend several chemicals to produce the repellent effect, or the repellent chemicals might be the constituents that were included in the feces in small amounts. Herbivores may recognize and avoid the compounds that are commonly contained in the feces of carnivore animals. If herbivore animals only avoid particular sympatric carnivore animals, there would be no explanation for the avoidance of sika deer to feces of allopatric lions in this study.

Although they were captive in the same facility, they had not met each other. Moreover, because the stockyard of sika deer was far from the breeding house of the lions, and there were many groves between them, it was not likely that the sika deer could smell the odor of lions. Chemicals from allopatric predators elicit defensive behavior of prey species (Weldon et al., 1993), and there was no significant difference between chemicals of predators that are sympatric or allopatric (Engelhart et al., 1995). Alternatively, prey species may similarly respond to several compounds.

Murray et al. (2006) reported that tiger fecal extract was effective in repelling goat feeding and the duration of the effectiveness was increased when encapsulated with bentonite. However, the effectiveness was only verified for three days. In contrast, our results showed that Fr.2 has the strongest effect and the effectiveness lasted at least throughout the four days of sample presentation in this study. Apfelbach et al. (2002) reviewed the effects of predator odors in mammalian prey species from both field and laboratory studies. Repellent effects may vary according to species of prey animal, and also depend on the types of behavioral control such as feeding suppression and passage deterrence.

In this study, it was indicated that the specific fraction separated from diethyl ether extracts of dried lion feces had the strongest repellent effects on sika deer, and the major constituents of the fraction by GC-MS analysis. The repellent chemicals could possibly be found in the unidentified compounds. Although the repellent chemicals were not identified in this study, additional purification might enable their identification. If the repellent chemicals could be identified and artificially synthesized, they may provide an effective repellent to deter passage of sika deer on railways.

\section{Acknowledgments}

We thank staff and T. Tsujimoto director of Morioka City Zoological Park for providing animals, the experimental pen, and lion fecal matter. We are grateful to JR-EAST Co., Ltd., Morioka branch for providing opportunity for this study. The study was partly supported by JR-EAST Co., Ltd., Morioka branch.

\section{References}

Andersen, K. F. and Vulpius, T. (1999). Urinary volatile constituents of the lion, Panthera leo. Chemical Senses, 24, 179-189.

Ando, C. (2003). The relationship between deer-train collisions and daily activity of the sika deer, Cervus nippon. Mammal Study, 28, 135-143.

Apfelbach, R., Blanchard, C. D., Blanchard, R. D., Hayes, R. A., Mcgregor, I. S. (2005). The effects of predator odors in mammalian prey species: A review of field and laboratory studies. Neuroscience and Biobehavioral Reviews, 29, 1123-1144.

Arnould, C., Malosse, C., Signoret, J.-P., Descoins, C. (1998). Which chemical constituents from dog feces are involved in its food repellent effect in sheep? Journal of Chemical Ecology, 24, 559-576.

Arnould, C., and Signoret, J. P. (1993). Sheep food repellents: Efficacy of various products, habituation, and social facilitation. Journal of Chemical Ecology, 19, 225-236.

Boag, B., Mlotkeiwicz, J.A. (1994). Effect of odor derived from lion feces on behavior of wild rabbits. Journal of Chemical Ecology, 20, 631-637.

Buesching, C. D., Waterhouse, J. P. and Macdonald, D. W. (2002a). Gas chromatographic analyses of the subcaudal gland secretion of the European badger (Meles meles); Part I: Chemical differences related to individual parameters. Journal of Chemical Ecology, 28, 41-56.

Buesching, C. D., Waterhouse, J. P. and Macdonald, D. W. (2002b). Gas-chromatographic analyses of the subcaudal gland secretion of the European badger (Meles meles) Part II: Time-related variation in the individual-specific composition. Journal of Chemical Ecology, 28, 57-69.

Burger, B. V., Visser, R., Moses, A. and Le Roux, M. (2006). Elemental sulfur identified in urine of cheetah, Acinonyx jubatus. Journal of Chemical Ecology, 32, 1347-1352. 
Burger, B. V., Viviers, M. Z., Bekker, J. P. I., Le Roux, M., Fish, N., Fourie, W. B. and Weibchen, G. (2008). Chemical characterization of territorial marking fluid of male Bengal tiger, Panthera tigris. Journal of Chemical Ecology, 34, 659-671.

Engelhart, A., Müller-Schwarze D. (1995). Responses of beaver (Castor canadiensis Kuhl) to predator chemicals. Journal of Chemical Ecology, 21, 1349-1364.

Goodrich, B. S., Heisterman, E.R., Shaw, K. S. and Mykytowycz, R. (1981). Identification of some volatile compounds in the odor of fecal pellets of the rabbit, Oryctolagus cuniculus. Journal of Chemical Ecology, $7,817-827$.

Kawamura. H. (2003). Research on the repellent effect of feces of carnivore on browsing damage on forest tree by sika deer. Tokushima Prefecture Forest and Forestry Research Institute Report, 2, 5-12 (in Japanese).

Kuroda K. and Honda T. (2004). Investigation of deterring method of deer feeding damage to nursery tree that uses lion feces. Yamanashi Prefecture Report (in Japanese).

Martin, J., Barja, I. and Lopez, P. (2010). Chemical scent constituents in feces of wild Iberian wolves (Canis lupus signatus). Biochemical Systematics and Ecology, 38, 1096-1102.

Mattina, M. J. I., Pignatello, J. J. and Swihart, R. K. (1991). Identification of volatile components of bobcat (Lynx rufus) urine. Journal of Chemical Ecology, 17, 451-462.

Murray, P. J., Burns, A.C., Davy, J.R. (2006). Development of an animal repellent-selection, efficacy and presentation. Australian Journal of Experimental Agriculture, 46, 851-856.

Ninomiya, S., Kabeda, N., Abe, N., and Sato, S. (2008). The aversive effect of wolf's faeces on Sika deer (Cervus nippon). Animal Behaviour and Management, 44, 215-219.

Preti, G., Muetterties, E. L., Furman, J. M., Kennelly, J. J. and Johns, B. E. (1976). Volatile constituents of dog (Canis familiaris) and coyote (Canis latrans) anal sacs. Journal of Chemical Ecology, 2, 177-186.

Rayer, J., Wiesler, D., Novotny M., Asa, C., Seal, U.S. and Mech, L.D. (1986). Chemical constituents in urine of wolf (Canis lupus) and their dependence on reproductive hormones. Journal of Chemical Ecology, 12, 297-314.

Sullivan, T.P., Nordstrom, L.O., and Sullivan, D.S. (1985). Use of predator odors as repellents to reduce feeding damage by herbivores II. Black-tailed deer (Odocoileus hemionus columbianus). Journal of Chemical Ecology, 11, 921-935.

Swihart, R. K., Pignatello, J. J., and Mattina, M. J. I. (1991). Aversive responses of white-tailed deer, Odocoileus virginianus, to predator urines. Journal of Chemical Ecology, 17, 767-777.

Seamans, T.W., Blackwell, B.F. and Cepek, J.D. (2002). Coyote hair as an area repellent for white-tailed deer. International Journal of Pest Management, 48, 301-306.

Weldon, P. J., Graham, D. P., and Mears, L. P. (1993). Carnivore fecal chemicals suppress feeding by alpine goats (Capra hircus). Journal of Chemical Ecology, 19, 2947-2952.

Wood, W. F. (2003). VOLATILE COMPONENTS IN METATARSAL GLANDS OF SIKA DEER, Cervus nippon. Journal of Chemical Ecology, 29, 2729-2733.

Wood, W. F., Terwilliger, M. N. and Copeland, J. P. (2005). Volatile compounds from anal glands of the wolverine, Gulo gulo. Journal of Chemical Ecology, 12, 2111-2117. 